\title{
Colour-texture mapping associations in terms of preference
}

\author{
Ela Fasllija $^{1}$ iD | Nilgün Olguntürk ${ }^{1}$ (D) | Dilek Güvenç ${ }^{2}$
}

\author{
${ }^{1}$ Department of Interior Architecture and \\ Environmental Design, Bilkent University, \\ Ankara, Turkey \\ ${ }^{2}$ Department of Mathematics, Bilkent \\ University, Ankara, Turkey \\ Correspondence \\ Ela Fasllija, Bilkent University, Ankara, \\ Turkey. \\ Email: e.fasllija@bilkent.edu.tr
}

\begin{abstract}
Basic design is the origin of many design-related fields and covers different concepts such as pattern, colour, texture and relief. Because colour is rarely seen as a uniformly plain element, current research embraces colour and texture as equally important variables. Investigations consider the association of colour and texture in terms of preference. One hundred individuals trained in design, as well as 96 individuals not trained in design, participated in an experiment in a virtual and abstract environment. We chose four primary colours from Natural Colour System colour space (yellow, red, green and blue) with the same saturation and brightness values. They were associated with three different texture types (plain, basket and broken twill), which we obtained by scanning the physical textile samples. In the second part of the experiment, we attempted to find a relationship between colour and one texture variable, in this case texture strength. The results of the study do not verify the existence of a dependency between colour and texture in either sample group. Also, there is no verification that a relationship between colour and texture strength exists. Furthermore, the results show that overall blue is the most preferred colour. Moreover, all participants preferred fine textures to coarse ones. Design-trained participants often associate fine strength level with basket texture whereas the non-design-trained participants more often associate it with plain textures. Lastly, the majority of participants state that blue is their most preferred colour in fine rather than coarse textures.
\end{abstract}

\section{1 | INTRODUCTION}

"To prefer" means to like the most and preferences are based on taste as well as on visual, tactile, auditory and olfactory signals. Visual aesthetics is considered to be the primary factor affecting preference. ${ }^{1}$ People show specific preferences towards different aspects of their surroundings; some prefer certain colours, and specific shapes, patterns and textures to others. ${ }^{2}$ In our surroundings, colour is rarely seen as an isolated element. More specifically, in interiors colour has a major impact on the quality of space, where most of the materials used have their own specific textures. "Non-uniformity of objects colours (texture) and their environment seems to be the rule rather than the exception (p. 426)."3

Colour and texture are complementary to each other in design fields. Especially in the textiles industry, the relationships between colour and texture have been widely studied. ${ }^{4-6}$
Colour affects the quality of fabric, ${ }^{4}$ but colour itself is also influenced by the texture of fabric. ${ }^{5-6}$ Texture can perceptually and instrumentally affect the colour of fabric. ${ }^{6}$

Researchers divide studies related to the effect of fabric texture on colour into three categories ${ }^{5}$ : the influence of texture on colour difference, the influence of texture on colour attributes and the relationship between texture descriptors and colours. This study pertains to the last group and attempts to find a relationship between colour and texture in terms of preference. There is a gap in knowledge in that there are not enough sources related to the association of these two variables in terms of preference. The majority of studies analyse the effect of texture on colour difference or colour attributes but none have explored the existence of a relationship between these factors. This study is concerned not only with texture type-colour association, but also with the texture strength (coarseness level)-colour association. 


\section{1 | Colour-texture related studies}

Kuehni and Marcus ${ }^{7}$ were the first researchers to investigate how the addition of texture affects colour perception. They evaluated object-colour samples according to their estimation and ranking, and concluded that the minimum value to perceive a difference between different texture types was one CIELab unit. Xin et $\mathrm{al}^{8}$ also studied the effect of texture and texture pattern in 15 woven textiles upon visual colour difference evaluation. Their findings supported the assumption that fabric samples have a significant impact on colour difference evaluation. Another study ${ }^{9}$ explored the effect of texture pattern on visual colour difference evaluation. The colour differences between textured and non-textured colour samples demonstrated that colour-texture-mapped pairs showed a reduction of 35\%-43\% in visual colour difference. The authors also found that increasing texture strength decreased the visual colour difference. In another study, Kandi and Tehran ${ }^{10}$ dyed eight different polyester textures with seven colours, red, orange, brown, yellow, green, blue and purple (each in five different concentrations of colour). The results indicated that texture structures have a significant effect upon colour difference. Han et $\mathrm{al}^{9}$ simulated fine and coarse textures in automobile coatings and found an insignificant impact of texture on colour differences in this particular context. These results may have turned out like this because of the specific context given to the study.

The addition of texture to a colour sample affects the colour's attributes. Shao et $\mathrm{al}^{11}$ explored the influence of texture structures of knitted fabrics on visual and instrumental colour and discovered that texture had a significant impact on the hue, chroma and lightness values of fabric colour. Montag and Berns ${ }^{12}$ revealed that textures increase lightness tolerance thresholds, and concluded that texture affects lightness tolerance, which in turn affects lightness thresholds. Another important finding of this study was that the impact of texture on the increase in lightness tolerance threshold was two times higher than that of regular stimuli. A similar study ${ }^{13}$ investigated the influence of texture on colour attribute tolerances. The authors used simulated random dot-textures on single colour samples and found that this type of texture influences the tolerances of lightness, chroma and hue.

Since texture has an impact on colour and its attributes, hue, saturation and brightness each have a major impact on colour perception and colour judgements. The earliest study to explore the effect of texture on the value of colours and their apparent warmth (preference) was conducted by Tinker ${ }^{14}$ in 1938 . He only used paper and cloth as his texture samples and did not find any significant effect of texture on the variables. One explanation for this may be a lack of available materials at that time.

Lucassen et al found that surface texture changed the emotional effect of coloured textile samples. ${ }^{3}$ Ten respondents were each asked to describe their emotional response to simulated colour chips in terms of four scales, warm-cool, masculine-feminine, hard-soft and heavy-light. In the first phase of the experiment, the participants had to rank solid colours according to the scales. Next, grayscale textures produced by using Perlin Noise were displayed on the computer monitor and were ranked horizontally. The textures produced for this part of the study were achromatic, differing only in lightness. In the last part of the experiment, colour and texture were mapped with each other and the respondents were asked once again to rank their emotional responses. The conclusion was that when texture was added to uniform colour samples, the respondents' emotional reactions to each colour changed. More specifically, the hard and soft scales of emotional responses were entirely determined by texture. ${ }^{3}$

In line with this study, it was found that adding texture can significantly influence the perceived unpleasantness of colours according to texture type. ${ }^{15}$ Even although this study was only concerned with only one type of emotion, it provides clues as to the importance of texture in colour samples.

The current study aims to find a relationship between two design elements, colour and texture, in terms of preference. The intention is to explore colour-texture-mapping associations according to visual preference for two different samples in a virtual environment as well as an abstract context.

\section{2 | METHODS}

\subsection{Sample groups}

To gain deeper insights into people's preferences two sample groups were recruited, each with a different background in terms of design training. Previous studies ${ }^{16,17}$ suggest that sample groups with different educational backgrounds exhibit contrasting approaches in terms of colour preference. An empirical study compared architects' and non-architects' evaluative and cognitive opinions and found that colour preference and the semantic rating of arousal and naturalness differed between the two groups. ${ }^{16}$ Furthermore, a study comparing the colour preferences of graphic design and information technology students with regard to living rooms verified that there is a significant relationship between educational background and colour preferences. ${ }^{17}$ In the current study, the design-trained sample group consisted of 100 participants (82 females and 18 males). Most of the respondents (71\%) in the first sample were sophomore students from the Interior Architecture and Environmental Design Department, Bilkent University. The others were from the Architecture, Graphic Design, Landscape Architecture and Communication and Design departments and of different grades. Ninety-six nondesign-trained participants (44 males and 52 females) also participated in this experiment. None of the participants 
in either sample group were colour blind according to the Ishihara Colour Blindness Test and they had no other vision or colour deficiencies preventing them from participating in the study.

\subsection{Experimental sets}

Nurus Design Office and Tepe Home Mobilya donated upholstery textile catalogues for use in this study. Because the saturation and brightness values between textures differed for the same colour, it was impossible to use these textile samples as physical experimental sets for the study.

Some studies ${ }^{5,6}$ have used an alternative but innovative way to produce realistic textile samples by scanning then displaying them in a virtual environment. This method eliminates the tactile sensation. This is relevant in that tactile sensation could influence the preferences stated by the participants in the current study. Firat Color studio scanned the neutral beige samples from the textiles in grayscale mode with the help of a Canon image FORMULA DR-F120 desktop scanner with 2400 dpi. The three scanned textile samples consisted of $100 \%$ polyester and each was one of the three textures used in the textiles industry, plain, basket or broken twill. ${ }^{6}$ The images were modified in Adobe Photoshop CS6 (RGB mode) with brightness and contrast values to achieve the same light grey colour (R: 214, G: 214, B: 214; Figure 1A-C).

The current study also examined texture strength. Two of the textures (plain and basket) have two texture strength levels, fine and coarse (Figure 2A,B). Broken twill has only one fine texture strength.

\section{3 | Colour-mapped sets}

Because of its basic and abstract context, this study used the four unique hues known as the psychological primaries (yellow, red, blue, green) as obtained from the Natural Colour System (NCS) catalogue. The saturation and brightness values were those which most closely resembled the physical samples. The NCS specifications are S2050-Y, S2050-R, S2050-G and S2050-B. By installing the NCS colour swatches in Adobe Photoshop, we were able to pick the chosen colour specifications using the
Colour Picker Tool. These were then mapped with the texture patches prepared previously. The $5 \times 5 \mathrm{~cm}$ images ( 4 colours $x 3$ textures $=12$ ) were placed on a neutral grey background ( $\mathrm{R}$ : 192, G: 192, B: 192). The same process was applied to the coarse texture samples in the second phase of the experiment. To reduce order effects, the squares' locations were changed randomly for every respondent according to a sequence obtained by random.org (Figures 3 and 4).

\section{$2.4 \quad$ The experiment}

The experiment was conducted in a science laboratory $\left(17.138 \mathrm{~m}^{2}\right.$ with white walls), which was ideal because it was windowless with controlled lighting. The environmental setup had cove lighting, which is suitable for experiments carried out in front of computer screens as it prevents glare. The room was lit with Standard Philips TL 54 fluorescent lights with a 6200 Kelvin colour temperature and 72-colour rendering index. The illuminance level was measured and fixed at 300 lx by a Minolta Illuminance meter.

In the experiment, the respondents were shown a 1366 x 768 pixels, 32-bit colour depth and $60 \mathrm{~Hz}$ refresh rate calibrated built-in (laptop) display. Twelve colour-texture-mapped squares were shown on a neutral grey background (Figure 3). The participants were seated $50 \mathrm{~cm}$ from the front of the monitor then asked to provide demographical information. Participants with visual deficiencies used spectacles or contact lenses to correct them. They were asked to carefully observe all 12 squares in the image then choose the square they preferred the most. If the first selected square had a plain or basket texture then the experiment proceeded to the second phase, when the preferred square (eg, plain blue) was shown in the same colour and texture but with two different texture strengths (eg, fine plain blue and coarse plain blue; Figure 4). The participant had to choose between two squares, the fine or coarse texture of their previously chosen colour-texture association. If, in the first phase of the experiment, a participant chose any colour associated with the broken-twill texture, then the experiment ended for that participant in the first phase and they were not included in the analysis related to colour-texture strength associations.
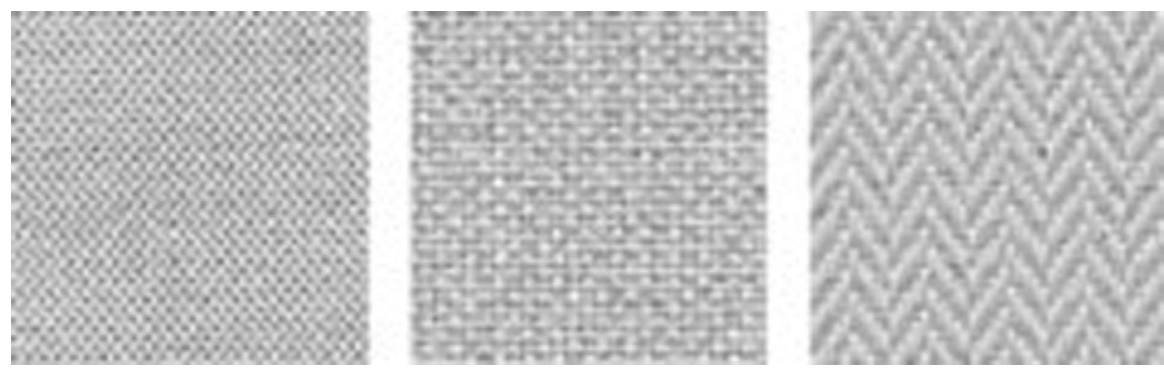

F IG URE 1 A, plain; B, basket; and C, broken twill 
FIGURE 2 A, plain coarse; and B, basket coarse

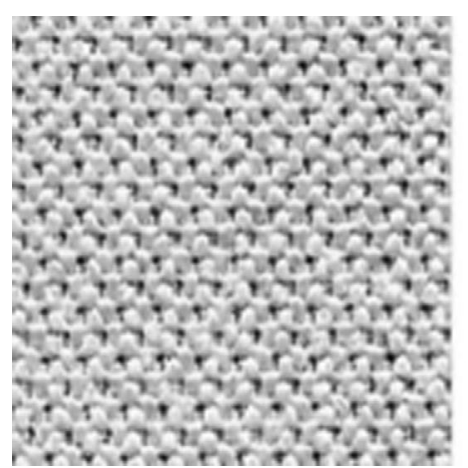

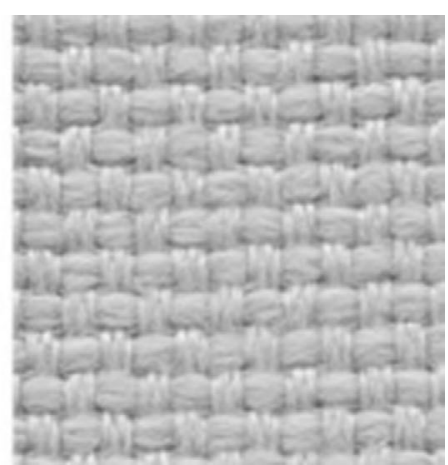

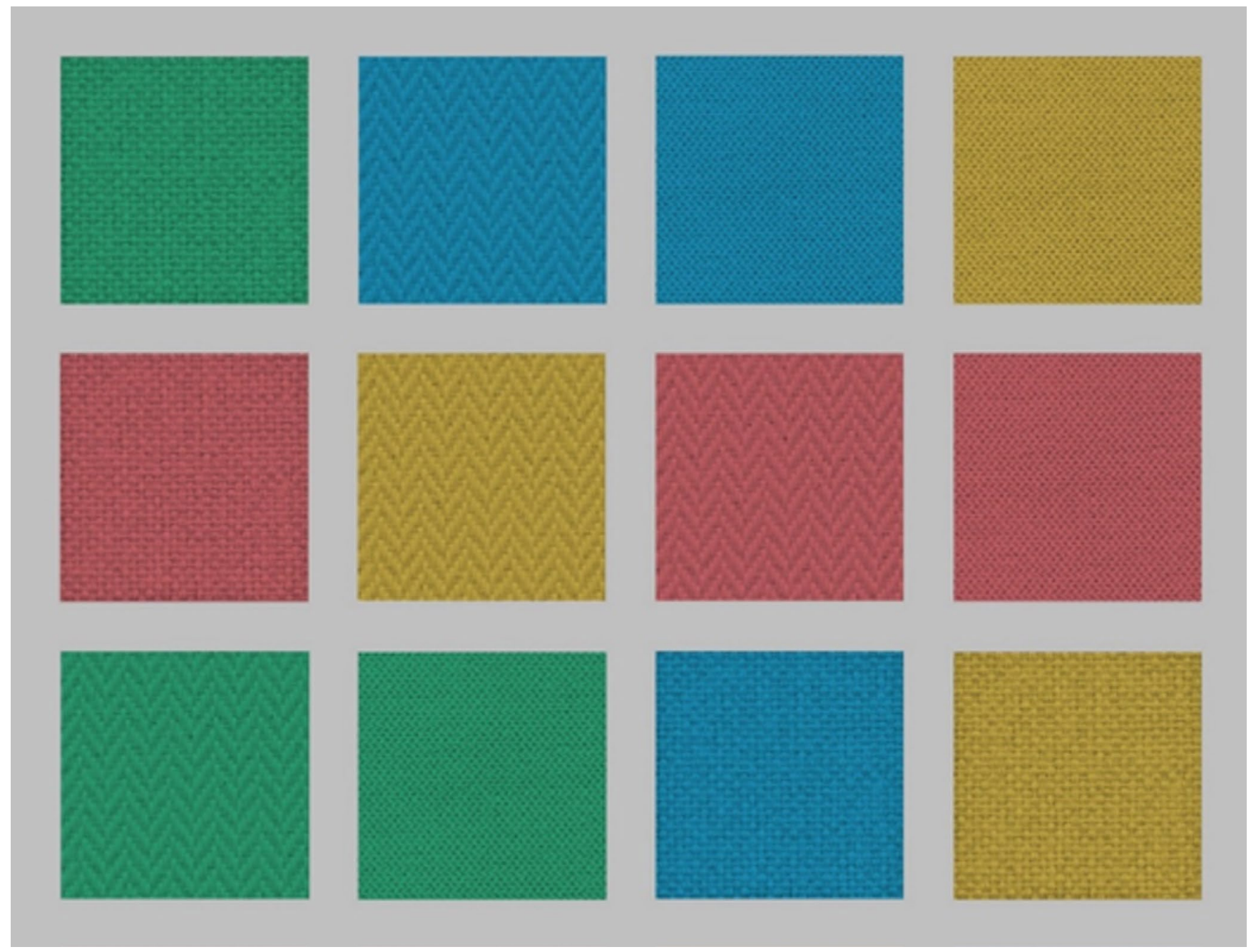

FI G URE 3 Texture-colour-mapped images [Colour figure can be viewed at wileyonlinelibrary.com]

\section{$3 \quad$ RESULTS}

\subsection{Colour and texture}

The data from the experiment were analysed using Statistical Package for the Social Sciences (SPSS) 20.0 software. To establish the existence of a possible dependency between the two main variables, colour and texture, we ran a Chi square test for independence. We then ran Chi square goodness of fit tests to determine if the distribution of colour and texture was equal between their respective categories. One-sample binomial tests were used to analyse colours, textures and texture strengths by comparing them in pairs.

Participants' colour-texture-mapped square preference frequencies are shown in Tables 1 and 2. Table 1 shows that $45 \%$ of participants chose the same basket texture irrespective of colour, and that $38 \%$ chose blue as their most preferred colour, irrespective of texture type. 

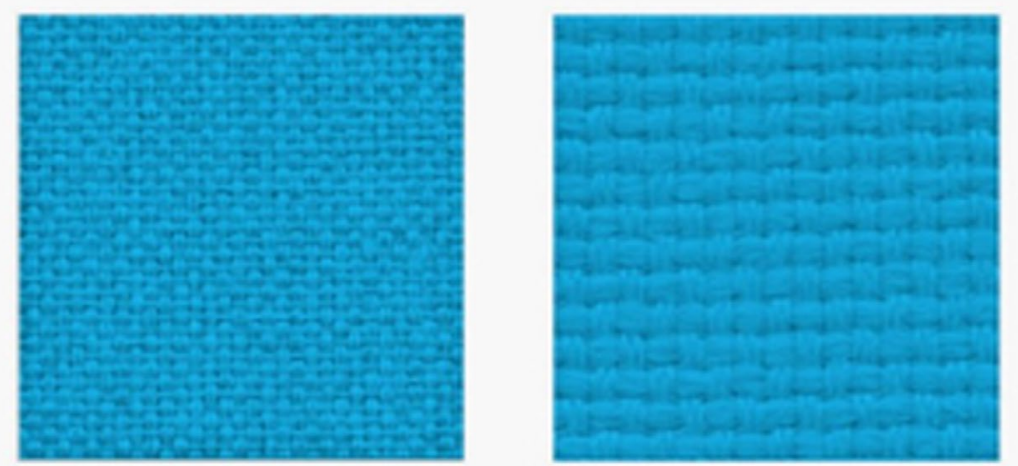

FIGURE 4 Fine coarse basket texture mapped with blue [Colour figure can be viewed at wileyonlinelibrary.com]

TA B L E 1 Colour-texture cross tabulation (design-trained)

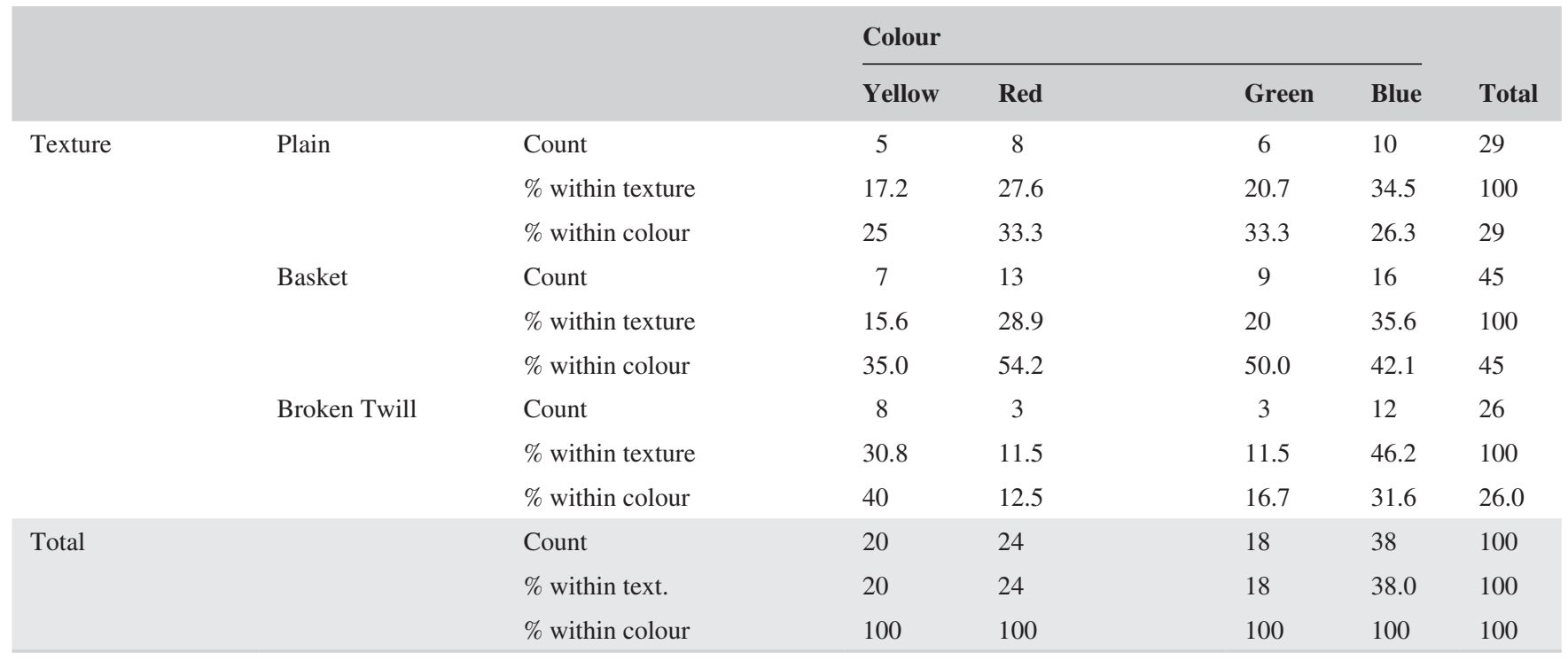

TA B L E 2 Colour-texture cross tabulation (non-design-trained)

\begin{tabular}{|c|c|c|c|c|c|c|c|}
\hline & & & \multicolumn{4}{|l|}{ Colour } & Total \\
\hline \multirow[t]{7}{*}{ Texture } & \multirow[t]{3}{*}{ Plain } & Count & 3 & 8 & 10 & 17 & 38 \\
\hline & & $\%$ within texture & 7.9 & 21.1 & 26.3 & 44.7 & 100 \\
\hline & & $\%$ within colour & 27.3 & 34.8 & 40 & 45.9 & 39.6 \\
\hline & Basket & $\%$ within colour & 36.4 & 34.8 & 24 & 37.8 & 33.3 \\
\hline & \multirow[t]{3}{*}{ Broken twill } & Count & 4 & 7 & 9 & 6 & 26 \\
\hline & & $\%$ within texture & 15.4 & 26.9 & 34.6 & 23.1 & 100 \\
\hline & & $\%$ within colour & 36.4 & 30.4 & 36 & 16.2 & 21 \\
\hline
\end{tabular}


In the second sample group (Table 2), the most preferred colour was again blue (38.5\%). The most preferred texture was plain (39.6\%). To establish if there was a dependency between the two main variables, we ran a Chi square test for independence for both sample sets and found that colour and texture are independent of each other in terms of preference. For the design-trained sample the results show that $\chi^{2}=5.788, d f=6, P=.447$ and $P>.05$; for the non-design-trained sample, $\chi^{2}=4.560$, $d f=6, P=.589$ and $P>.05$. These findings reject the first hypothesis of this study, which states that a dependency between the variables exists. Moreover, a Chi square goodness of fit test explored whether different textures would have resulted in different preference rankings and revealed that it was only in the design-trained sample group that texture types were not preferred equally $\left(\chi^{2}\right.$ $(2)=6.260, P=.044, P<.05)$. The binomial one-sample test between texture types showed that the basket texture was preferred to broken twill $(P=.033)$.

Previous studies ${ }^{18,19}$ suggest that blue is the preferred colour in any context. A goodness of fit Chi square test was conducted to discover whether colour categories (yellow, red, green, blue) are equally preferred by the participants in both sample groups. It was found that different colours were not preferred equally by participants in the design and non-design-trained groups $\left(\left[\chi^{2}(3)=9.760, P=.021, \mathrm{p}<0.05\right]\right.$ and $\left[\chi^{2}=14.167, d f=3, P=.003, P<.005\right]$, respectively). The binomial one-sample tests found that blue was more preferred than yellow $(P=.026)$ or green $(P=.011)$ in the first sample group.

\section{2 | Colour and texture strength}

Tables 3 and 4 show the choices participants made in the second phase of the experiment. Seventy-four of 100 designtrained participants chose those textures which had two texture strengths.

\section{3 | Texture strength preference}

The results show that there was no significant association between colour and texture strength in terms of preference in the design and non-design-trained groups $\left(\left[\chi^{2}(3)=3.481\right.\right.$, $P=.323, P>.005]$ and $\left[\chi^{2}=0.641 d f=3, P=.887\right.$, $P>$.05], respectively). To establish if there was a more preferred texture strength irrespective of texture type and colour, a one-sample binomial test was run. $P$-values of .004 (designtrained) and $P=.000$ (non-design-trained), both of which are less than the significance level, show that fine textures were preferred over coarse ones.

Moreover, in the non-design-trained set, the results from the binomial test showed that fine plain textures were preferred to coarse plain textures $(P=.000)$. For basket textures, fine strengths are more often preferred in both sets $(P=.037 ; P=.017)$. We ran extra tests to ascertain if a particular texture strength was preferred within a specific colour. The results showed that red fine textures were preferred more than red coarse textures in the non-designtrained group $(P=.21)$. Finally, all of the participants preferred fine blue textures to coarse textures $(P=.011$; $P=.027)$.

\section{DISCUSSION AND CONCLUSIONS}

Based on the literature, it was expected that individuals who indicated a specific preference in one domain would state a similar preference in another domain. ${ }^{2}$ This study aimed to find an association between colour and texture as they overlapped with one another. Typically, in colour studies, colour is studied within its attributes. In the current study, colour dimensions (apart from hue) remained constant. The changing variable was texture strength. For two textures, two different texture strengths were used and analysed in terms of preference. Specifically, plain and basket had fine and coarse
T A B L E 3 Colour-texture strength cross tabulation (design-trained)

\begin{tabular}{|c|c|c|c|c|c|c|c|}
\hline & & & \multicolumn{4}{|l|}{ Colour } & \multirow[b]{2}{*}{ Total } \\
\hline & & & Yellow & Red & Green & Blue & \\
\hline \multirow[t]{6}{*}{ Strength } & \multirow[t]{3}{*}{ Fine } & Count & 8 & 11 & 11 & 20 & 50 \\
\hline & & $\%$ within texture & 16 & 22 & 22 & 40 & 100 \\
\hline & & $\%$ within colour & 66.7 & 52.4 & 73.3 & 76.9 & 29 \\
\hline & \multirow[t]{3}{*}{ Coarse } & Count & 4 & 10 & 4 & 6 & 24 \\
\hline & & $\%$ within texture & 16.7 & 41.7 & 16.7 & 25 & 100 \\
\hline & & $\%$ within colour & 33.3 & 47.6 & 26.7 & 23.1 & 32.4 \\
\hline \multirow[t]{3}{*}{ Total } & & Count & 12 & 21 & 15 & 26 & 74 \\
\hline & & $\%$ within texture & 16.2 & 28.4 & 20.3 & 35.1 & 100 \\
\hline & & $\%$ within colour & 100 & 100 & 100 & 100 & 100 \\
\hline
\end{tabular}




\begin{tabular}{|c|c|c|c|c|c|c|c|}
\hline & & & \multicolumn{4}{|l|}{ Colour } & \multirow[b]{2}{*}{ Total } \\
\hline & & & Yellow & Red & Green & Blue & \\
\hline \multirow[t]{6}{*}{ Strength } & \multirow[t]{3}{*}{ Fine } & Count & 6 & 13 & 12 & 16 & 47 \\
\hline & & $\%$ within texture & 12.8 & 27.7 & 25.5 & 34 & 100 \\
\hline & & $\%$ within colour & 85.7 & 81.3 & 75 & 76.2 & 78.3 \\
\hline & \multirow[t]{3}{*}{ Coarse } & Count & 1 & 3 & 4 & 5 & 13 \\
\hline & & $\%$ within texture & 7.7 & 23.1 & 30.8 & 38.5 & 100 \\
\hline & & $\%$ within colour & 14.3 & 18.8 & 25 & 23.8 & 21.7 \\
\hline \multirow[t]{3}{*}{ Total } & & Count & 7 & 16 & 15 & 21 & 60 \\
\hline & & $\%$ within texture & 11.7 & 26.7 & 26.7 & 35.0 & 100 \\
\hline & & $\%$ within colour & 100 & 100 & 100 & 100 & 100 \\
\hline
\end{tabular}

T A B L E 4 Colour-texture strength cross tabulation (non-design-trained) texture levels. They were also mapped with the chosen colours of yellow, red, blue and green.

The first hypothesis of the study (stating the existence of a dependency between colour and texture) was rejected in both groups. Even although accumulated evidence shows that individual preferences for different domains are not independent of each other, the domains (colour and texture) overlapped one another. The methodology of the study did not consist of showing colours and textures separately, but instead these were mapped with each other, which is why the preference stated was a result of the combination of texture and colour. It is known, especially in the case of fabrics, that the combination of colour and texture affects consumers' preferences. ${ }^{20}$ If colour chips were shown first, and non-coloured textures second, perhaps a cross-preference between the two domains would then exist. This method would still not be accurate enough to evaluate colour-texture associations because of the influence they have on each other. We examined participants only according to their age, gender and background, but many other factors can affect individual preferences. Another factor that may affect this result is the number of the participants. The analysis test (Chi square test for independence) requires a large sample set. This study also assumes that one texture will be preferred over the others, which was verified in the design-trained sample set. This occurs because, as the literature suggests, the sample set is composed of individuals who are more sensitive to detail. ${ }^{18}$ Apart from choosing colours, they paid more attention to texture choice. This study's findings agree with other researchers who reported that overall blue is the favourite colour. ${ }^{18-19,21-23}$ Chi square tests show an unequal distribution of responses in colour preferences. Blue has the highest preference percentage in both sets.

Next, we probed for possible associations between colour and texture strength. Because previous studies proved that texture strength has a significant impact on the lightness values of colour, ${ }^{12}$ and because lightness also influences colour preferences, ${ }^{24}$ it was deduced that a specific texture strength would be preferred over another. We found no dependency between colour and texture strength in either set, but we did verify the hypothesis which assumes that one texture strength is preferred over another: fine textures were preferred to coarse textures. Furthermore, the texture strength preference for each colour was investigated. Because blue had the largest frequency distribution, it was the only colour with an association between the blue hue and fine textures for all of the participants. The same occurred for basket texture type and fine strength. In this case, it can be inferred that a large number of responses yields more accurate statistical results.

This study also has other limitations. Using physical textile samples might have been interesting in terms of texture effects. Even although the study was only concerned with the visual effect of texture, tactile sensation could provide further data. Nevertheless, conducting this study in a virtual environment was more feasible in terms of time and costs. ${ }^{6}$ Also, designers are used to working in virtual environments and do not experience difficulties in translating simulated works into realistic ones. Supporting this statement, Suk and $\operatorname{Irtel}^{25}$ found that media type (physical vs simulated) had no effect on emotional responses to colour.

It should be noted that this study's results are neither definitive nor prescriptive due to a variety of confounding factors that affect individual preferences. However, it is of value for design professionals seeking to gain an insight into colour-texture combinations. Manufacturers could also benefit by understanding what the most preferred colour-texture matches are.

Because this was a preliminary study, it offers several possibilities for future research. Further work could investigate colour-texture association preferences in a physical environment. Additionally, more colours and textures, or perhaps even the combination of two colours with two textures, could be investigated under the concept of colour-texture harmony. The most used colours in upholstery fabrics (such as brown and beige) could also be chosen for future study. More fabric types and more texture strength levels could also be used. 
It would be practical for future studies to provide new insights into the experimental methodology. Another approach would be to show the colour-texture-mapped images one by one and to ask for each user's preference rating. This would give mean data then more statistical tests could then be conducted. But preferring is different to liking. To have a preference is to like one alternative more than another (or others). Ranking the images from the most to the least preferred could be another way to examine the relationships we are interested in. This would give median values. On the other hand, using a lot of variables (more colours and textures) could result in the data becoming too complicated to understand.

To secure "universal" preference results, a large number of people should participate in the experiment. Additionally, because texture is present not only in fabrics but also in other architectural materials, one could consider using these in other studies. People can have a different combination of preferences for curtains or for carpets. The investigation of preferences for all of these topics would provide better information for architects to design spaces that users will enjoy. Design is not an exact field of study; nevertheless, architects tend to have "universal standards" in terms of design harmony, in order to produce quality spaces for their clients.

\section{ORCID}

Ela Fasllija (iD https://orcid.org/0000-0002-5631-029X

Nilgün Olguntürk (iD https://orcid.

org/0000-0001-7947-1101

\section{REFERENCES}

1. Yi E, Choi J. Intergenerational differences of color sensation and preference for naturally dyed fabrics. Fibers Polym. 2008;9:646-652.

2. Chen N, Tanaka K, Matsuyoshi D, Watanabe K. Correlated preferences for color and shape. Color Res Appl. 2016;41:188-195.

3. Lucassen M, Gevers T, Gijsenij A. Correlated preferences for color and shape. Color Res Appl. 2011;36:426.

4. Luo L, Tsang K, Shen H, Shao S, Xin J. An investigation of how the texture surface of a fabric influences its instrumental color. Color Res Appl. 2015;40:472-482.

5. Xin J, Shen H, Lam C. Investigation of texture effect on visual color difference evaluation. Color Res Appl. 2005;30:341-347.

6. Kandi S, Tehran M, Rahmati M. Colour dependency of textile samples on the surface texture. Color Technol. 2008;124:348-354.

7. Kuehni RG, Marcus RT. An experiment in visual scaling of small color differences. Color Res Appl. 1979;4:83.

8. Xin JH, Chuen LC, Luo MR. Congress of the International Colour Association, (AIC03rd-8th). Bangkok, Thailand: 2003:60.
9. Han B, Cui GH, Luo MR. Congress of the International Colour Association, (AIC03rd-8) vol. 176. Bangkok, Thailand: 2003.

10. Kandi S, Tehran M. Color naming for the Persian language. Color Res Appl. 2010;35:94.

11. Shao SJ, Xin J, Zhang YG, Zhou LM. The effect of texture structure on instrumental color difference evaluation and visual assessment. AATCC Rev. 2006;6:42.

12. Montag E, Berns R. Lightness dependencies and the effect of texture on suprathreshold lightness tolerances. Color Res Appl. 2000;25:241-249.

13. Huertas R, Melgosa M, Hita E. Influence of random-dot textures on perception of suprathreshold color differences. J Opt Soc Am. 2006;23:2067-2076.

14. Tinker MA. Effect of stimulus-texture upon apparent warmth and affective values of colors. Am J Psychol. 1938;51:532-535.

15. Simmons D, Russell C. Visual texture affects the perceived unpleasantness of colors. Perception. 2008;37:146.

16. Cubukcu E, Kahraman I. Hue, saturation, lightness, and building exterior preference: an empirical study in Turkey comparing architects' and nonarchitects' evaluative and cognitive judgments. Color Res Appl. 2008;33:395-405.

17. Hanafy I, Sanad R, Miljkovic D.6th World Conference on Psychology, Counseling and Guidance (Wcpcg-2015), 2015;205:437.

18. Camgoz N, Yener C, Guvenc D. Effects of hue, saturation, and brightness on preference. Color Res Appl. 2002;27:199-207.

19. Bakker I, van der Voordt T, Vink P, de Boon J, Bazley C. Color preferences for different topics in connection to personal characteristics. Color Res Appl. 2015;40:62-71.

20. Larsen JL, Weeks JG. Fabrics For Interiors: A Guide for Architects, Designers, and Consumers. New York, NYVan Nostrand Reinhold Co; 1975 .

21. Eysenck HJ. A critical and experimental study of colour preferences. Am J Psychol. 1941;54:385-394.

22. Manav B. Color-emotion associations and color preferences: a case study for residences. Color Res Appl. 2007;32:144-150.

23. Baniani M, Yamamoto S. A comparative study on correlation between personal background and interior color preference. Color Res Appl. 2015;40:416-424.

24. Guilford JP, Smith P. A system of color-preferences. Am J Psychol. 1959;62:487-502.

25. Suk H, Irtel H. Emotional response to color across media. Color Res Appl. 2010;35:64-77.

How to cite this article: Fasllija E, Olguntürk N, Güvenç D. Colour-texture mapping associations in terms of preference. Coloration Technol. 2020;136:468-475. https://doi.org/10.1111/cote.12492 